\title{
Erratum to: mGluR2/3 agonist LY379268 rescues NMDA and GABAA receptor level deficits induced in a two-hit mouse model of schizophrenia
}

\author{
Martin Engel ${ }^{1,2,3,4} \cdot$ Peta Snikeris ${ }^{1,2,3} \cdot$ Natalie Matosin ${ }^{1,2,3}$. \\ Kelly Anne Newell ${ }^{1,2,3} \cdot$ Xu-Feng Huang ${ }^{1,2,3}$ • Elisabeth Frank ${ }^{1,3}$
}

Published online: 9 March 2016

(C) Springer-Verlag Berlin Heidelberg 2016

\section{Erratum to: Psychopharmacology \\ DOI 10.1007/s00213-016-4230-0}

Legends of Figs. 1, 2 and 3 were misplaced in the original version of this article. Correct legends are presented below:

Fig. 1 Acute LY379268 and olanzapine treatment restore NMDA-R binding density levels in several brain regions of two-hit NRG1 HET (PCP) mice. Wild type mice (WT) and neuregulin 1 heterozygous transmembrane domain knockout mice (HET) received chronic phencyclidine (PCP) or saline (SAL) treatment for 14 days and an acute treatment of LY379268 or olanzapine after 14 days washout. N-methyl$\mathrm{D}$-aspartate receptors (NMDA-R) binding was quantified in the prefrontal cortex (PFC), caudate putamen $(\mathrm{CPu})$, Nucleus

The online version of the original article can be found at http://dx.doi.org/ 10.1007/s00213-016-4230-0.

Martin Engel

mengel@uow.edu.au

Schizophrenia Research Institute, Sydney, Australia

2 Faculty of Science Medicine and Health, University of Wollongong, Wollongong, Australia

3 Illawarra Health and Medical Research Institute, University of Wollongong, Wollongong, Australia

4 School of Biological Sciences, University of Wollongong, Wollongong, Australia accumbens (NAcb), whole hippocampus (Hipp) and subregions (CA1), dentate gyrus (DG), lateral septum (LS). Data presented as mean binding density $\mathrm{nCi} / \mathrm{mg}$ tissue \pm standard error of the mean $(n=6)$, Statistical significance: ${ }^{*} P<0.05$, ** $P<0.01,{ }^{* * *} P<0.001$ as compared to $\mathrm{WT}(\mathrm{SAL})$ vehicle

Fig. 2 Acute LY379268 and olanzapine treatment restore GABAA-R binding density levels in several brain regions of two-hit NRG1 HET (PCP) mice. Wild type mice (WT) and neuregulin 1 heterozygous transmembrane domain knockout mice (HET) received chronic phencyclidine (PCP) or saline (SAL) treatment for 14 days and an acute treatment of LY379268 or olanzapine after 14 days washout. Gammaaminobutyric acid receptor A (GABAA-R) binding was quantified in the prefrontal cortex (PFC), caudate putamen $(\mathrm{CPu})$, Nucleus accumbens (NAcb), whole hippocampus (Hipp) and subregions (CA1), dentate gyrus (DG), lateral septum (LS). Data presented as mean binding density $\mathrm{nCi} / \mathrm{mg}$ tissue \pm standard error of the mean $(n=6)$, Statistical significance: $* P<0.05$, ${ }^{* *} P<0.01,{ }^{* * *} P<0.001$ as compared to $\mathrm{WT}(\mathrm{SAL})$ vehicle

Fig. 3 Acute LY379268 treatment restores positive correlation between NMDA-R and GABAA-R binding levels across brain regions in two-hit NRG1 HET(PCP) mice. Spearman's correlation plots depicting the relationship between NMDA-R and GABAA-R binding levels across all brain regions, following the different modelling strategies and acute treatment options. Abbreviations: wild type mice (WT), neuregulin 1 heterozygous transmembrane domain knockout mice (NRG1), chronic phencyclidine treatment (PCP), chronic saline treatment $(\mathrm{SAL}) * P>0.05, * * P>0.01$ 